\title{
ALEXANDER MODULES
}

\author{
NOBUYUKI SATO
}

\begin{abstract}
The Alexander modules of a link are the homology groups of the universal abelian cover of the complement of the link. For a link of $n$-spheres in $S^{n+2}$, we show that, if $n \geq 2$, the Alexander modules $A_{2}, \ldots, A_{n}$ and the torsion submodule of $A_{1}$ are all of type $L$. This leads to a characterization, below the middle dimension, of the polynomial invariants of the link. These results were previously proven for the special case of boundary links.
\end{abstract}

The purpose of this note is to give a simplified proof of a generalization of one of the principal results of [Sa]. In that work, we showed that the Alexander modules of a higher-dimensional boundary link are of a certain kind, called type L. As a corollary, we were able to characterize the polynomial invariants of a higherdimensional boundary link, below the middle dimension. Here, we prove the results for all higher-dimensional links.

Some of the results in this paper can be obtained from work of Dwyer [Dw] or Smith [Sm]. Lemma 1 is a direct consequence of a result of Lichtenbaum [Li]. However, we will give a unified treatment via finite free resolutions [BE, BE2].

The main result of this paper was claimed in $[\mathbf{S a}]$ in a remark. We apologize for the delay.

Finally, the author wishes to thank Sue Geller for helpful conversations.

1. Modules of type $L$. Let $\Lambda$ be the integral group ring of the free abelian group of rank $m$. We may identify $\Lambda$ with the ring $\mathbf{Z}\left[x_{1}^{ \pm 1}, x_{2}^{ \pm 1}, \ldots, x_{m}^{ \pm 1}\right]$ of Laurent polynomials. Thus, $\Lambda$ is a commutative, noetherian domain with global (homological) dimension $m+1$, over which all finitely generated projective modules are free (see [Sw]). If $M$ is a finitely generated $\Lambda$-module, we say that $M$ is of type $L$ if $\mathbf{Z} \otimes_{\Lambda} M=$ 0 . Here, $\mathbf{Z}$ is regarded as a $\Lambda$-module via the augmentation homomorphism $\epsilon: \Lambda \rightarrow$ Z.

We now state the main algebraic result of this paper. Let

$$
\mathbf{F}: F_{2} \stackrel{\phi_{2}}{\rightarrow} F_{1} \stackrel{\phi_{1}}{\rightarrow} F_{0}
$$

be a complex of free $\Lambda$-modules of finite rank, and denote by $\mathbf{Z} \otimes \mathbf{F}$ the complex of free abelian groups obtained by tensoring the complex $\mathbf{F}$ with $\mathbf{Z}$ over $\Lambda$.

Theorem A. Suppose that $H_{1}(\mathbf{Z} \otimes \mathbf{F})=0$. Then $H_{1}(\mathbf{F})$ is of type $L$.

The proof of this result depends on a small portion of the theory of finite free resolutions (see [BE, BE2]). For the convenience of the reader, we will summarize the aspects we need.

Received by the editors June 16, 1983.

1980 Mathematics Subject Classification. Primary 57Q45, 18G15.

Key words and phrases. Alexander module, link module. 
Let $R$ be a commutative noetherian ring with unit, and let $f: G \rightarrow H$ be a homomorphism of finitely generated free modules. A choice of bases for $G$ and $H$ allows us to associate a matrix to $f$. For each positive integer $i$, let $U_{i}$ be the ideal in $R$ generated by the $i \times i$ minor determinants of this matrix. We set $U_{0}=R$, and for $i$ sufficiently large, $U_{i}=0$ because there are no $i \times i$ minors. The $U_{i}$ form a decreasing sequence. The rank of $f$, denoted $\operatorname{rk}(f)$, is the largest integer $k$ for which $U_{k} \neq\{0\}$. For $k=\operatorname{rk}(f)$, we set $I(f)=U_{k}$. The sequence $U_{i}$ depends only on $f$.

The depth of an ideal $J$ of $R$ is the length of any maximal $R$-sequence formed from elements of $J$. For $J \neq R$, this is a well-defined integer. If $J=R$, we say $\operatorname{depth}(J)=\infty$. The radical of $J$, denoted $\operatorname{Rad}(J)$, is the set of all $x$ such that some power of $x$ is in $J$. We will use the following facts. Let

$$
\mathbf{F}: 0 \rightarrow F_{n} \stackrel{\phi_{n}}{\rightarrow} F_{n-1} \rightarrow \cdots \rightarrow F_{1} \stackrel{\phi_{1}}{\rightarrow} F_{0}
$$

be a complex of free $R$-modules of finite rank.

(I) [BE, Corollary 1] $\mathbf{F}$ is exact if and if for each $k, \operatorname{rk}\left(\phi_{k}\right)+\operatorname{rk}\left(\phi_{k+1}\right)=\operatorname{rk}\left(F_{k}\right)$ and $\operatorname{depth}\left(I\left(\phi_{k}\right)\right) \geq k$.

(II) [BE2, Theorem 2.1] If $\mathbf{F}$ is exact, then for all $k, \operatorname{Rad}\left(I\left(\phi_{k}\right)\right) \subseteq \operatorname{Rad}\left(I\left(\phi_{k+1}\right)\right)$.

Note that if $R=\mathbf{Z}$ in (I), the depth condition translates to the requirement that $I\left(\phi_{k}\right)=\mathbf{Z}$ for all $k>1$.

LEMMA 1. Let $M$ be a finitely generated $\Lambda$-module such that $\operatorname{Tor}_{k}^{\Lambda}(\mathbf{Z}, M)=0$ for some $k \geq 0$. Then $\operatorname{Tor}_{j}^{\Lambda}(\mathbf{Z}, M)=0$ for all $j \geq k$.

Remarks. The case $k=0$ was proved independently in [Dw, Sa and Sm]. This lemma is also a consequence of $[\mathbf{L i}]$.

Proof. Choose a finite free resolution for $M$. This is a resolution of finite length $\left\{F_{k}, \phi_{k}\right\}$ such that each $F_{k}$ is free of finite rank. Such a resolution always exists, since $\Lambda$ is noetherian of finite global dimension and all finitely generated projectives are free [Sw]. If $\operatorname{Tor}_{k}^{\Lambda}(\mathbf{Z}, M)=0$, then $\mathbf{Z} \otimes_{\Lambda} F_{k+1} \rightarrow \mathbf{Z} \otimes_{\Lambda} F_{k} \rightarrow \mathbf{Z} \otimes_{\Lambda} F_{k-1}$ is exact (if $\left.k=0, F_{k-1}=0\right)$. By (I) above, $I\left(1 \otimes \phi_{k+1}\right)=\mathbf{Z}$. Since $\epsilon$ is onto, $\epsilon\left(I\left(\phi_{k+1}\right)\right)=I\left(1 \otimes \phi_{k+1}\right)$. However, by (II), $\operatorname{Rad}\left(\phi_{i}\right)$ form an increasing sequence; hence $\epsilon\left(I\left(\phi_{j}\right)\right)=I\left(1 \otimes \phi_{j}\right)=\mathbf{Z}$ for all $j>k$. This implies that $\operatorname{rk}\left(1 \otimes \phi_{j}\right)=\operatorname{rk}\left(\phi_{j}\right)$ for all $j>k$. We now apply (I) to conclude that $\operatorname{Tor}_{j}^{\Lambda}(\mathbf{Z}, M)=0$ for all $j>k$.

Proof of THEOREM A. There is a universal coefficient spectral sequence for homology (see, for example, $[\mathbf{H W}]$ ) with $E^{2}$-term given by $E_{p, q}^{2}=\operatorname{Tor}_{p}^{\Lambda}\left(\mathbf{Z}, H_{q}(\mathbf{F})\right.$ ) and differential of degree $(-r, r-1)$ converging to $H_{*}(\mathbf{Z} \otimes \mathbf{F})$. Since $H_{1}(\mathbf{Z} \otimes \mathbf{F})=0$ by hypothesis, we must have $E_{0,1}^{\infty}=E_{1,0}^{\infty}=0$. However, $E_{1,0}^{\infty}=E_{1,0}^{2}=\operatorname{Tor}_{1}^{\Lambda}\left(\mathbf{Z}, H_{0}(\mathbf{F})\right)$ and $E_{0,1}^{\infty}$ is the cokernel of $d_{2,0}^{2}: \operatorname{Tor}_{2}^{\Lambda}\left(\mathbf{Z}, H_{0}(\mathbf{F})\right) \rightarrow \mathbf{Z} \otimes_{\Lambda} H_{1}(\mathbf{F})$. Since $\operatorname{Tor}_{1}^{\Lambda}\left(\mathbf{Z}, H_{0}(\mathbf{F})\right)$ $=0$, we can apply Lemma 1 to see that $\operatorname{Tor}_{2}^{\Lambda}\left(\mathbf{Z}, H_{0}(\mathbf{F})\right)=0$. But then $d_{2,0}^{2}$ is a homomorphism of 0 onto $\mathbf{Z} \otimes_{\Lambda} H_{1}(\mathbf{F})$. Hence, $H_{1}(\mathbf{F})$ is of type $L$.

2. Alexander modules. Let $X$ be the complement of an $m$-component link of $n$-spheres in $S^{n+2}, n \geq 2$. By Alexander duality, $H_{1}(X) \approx \mathbf{Z}^{m}$. If $\tilde{X}$ is the universal abelian cover of $X$, then the homology groups of $\tilde{X}$ are finitely generated $\Lambda$-modules. These modules are called the Alexander modules of the link.

THEOREM B. Let $\tilde{X}$ be the universal abelian cover of the complement $X$ of a link of $n$-spheres in $S^{n+2}, n \geq 2$. Then:

(i) $H_{q}(\tilde{X})$ is a $\Lambda$-module of type $L$ for $2 \leq q \leq n$.

(ii) If $T_{1}$ is the $\Lambda$-torsion submodule of $H_{1}(\tilde{X})$, then $T_{1}$ is of type $L$. 
REMARK. We may now use results of [Sa] to completely characterize the polynomial invariants of a higher-dimensional link, below the middle dimension (see [Sa] for this characterization).

Part (i) of Theorem B follows directly from Theorem A, since $H_{q}(X)=0$ for $2 \leq q \leq n$. Part (ii) requires a more circuitous approach.

Let $F_{m}$ denote the free (nonabelian) group on $m$ letters, and let $\pi=\pi_{1}(X)$. There is a homomorphism of $F_{m}$ to $\pi$ which carries the generators to meridians of the link. It follows from results of Kervaire [Ke] and Stallings [St] that this is a monomorphism, which induces an isomorphism on $H_{1}$. Let $V$ be the $m$-fold wedge of circles. The homomorphism $F_{m} \rightarrow \pi$ is realized by a mapping of $V$ into $X$, which we can regard as an inclusion. Since the inclusion induces an identification on $H_{1}$, we obtain an equivariant map $i: \tilde{V} \rightarrow \tilde{X}$. Thus, $i_{*}: H_{*}(\tilde{V}) \rightarrow H_{*}(\tilde{X})$ is a homomorphism of $\Lambda$-modules.

\section{LEMMA 2. $i_{*}$ induces an isomorphism}

$$
1 \otimes i_{*}: \mathbf{Z} \otimes_{\Lambda} H_{1}(\tilde{V}) \rightarrow \mathbf{Z} \otimes_{\Lambda} H_{1}(\tilde{X}) .
$$

In particular, if $K=\operatorname{cok} i_{*}$, then $K$ is of type $L$.

Proof. The equivariant map $i$ induces a homomorphism of spectral sequences of the coverings $\tilde{V} \rightarrow V$ and $\tilde{X} \rightarrow X$. At the $E^{2}$-level, we have a commuting diagram

$$
\begin{array}{ccc}
H_{0}\left(\mathbf{Z}^{m} ; H_{1}(\tilde{V})\right) & \rightarrow & H_{0}\left(\mathbf{Z}^{m} ; H_{1}(\tilde{X})\right) \\
\uparrow & & \uparrow \\
H_{2}^{m}\left(\mathbf{Z}, H_{0}(\tilde{V})\right) & \rightarrow & H_{2}^{m}\left(\mathbf{Z} ; H_{0}(\tilde{X})\right)
\end{array}
$$

where the horizontal maps are induced by $i$ and the vertical maps are the $E^{2}$ differentials. The bottom map is clearly an isomorphism. The vertical maps are isomorphisms as well; this follows from the fact that $H_{1}(V) \approx H_{1}(X) \approx \mathbf{Z}^{m}$ and an argument similar to that given in the proof of Lemma 1. Hence, all four maps are isomorphisms. Since $H_{0}\left(\mathbf{Z}^{m} ; M\right)=\mathbf{Z} \otimes_{\Lambda} M$ for any $\Lambda$-module $M$, the lemma follows. The statement about $K$ follows from the right-exactness of tensor product.

Any module of type $L$ is a $\Lambda$-torsion module. This is because $\mathbf{Z} \otimes_{\Lambda} M \approx M / A M$, where $A=\operatorname{ker}\{\epsilon: \Lambda \rightarrow \mathbf{Z}\}$ is the ideal generated by $\left\{x_{1}-1, \ldots, x_{m}-1\right\}$ in $\Lambda$. Nakayama's lemma applies to show that if $Z \otimes_{\Lambda} M=0$, then $M$ is annihilated by an element of $\Lambda$ of the form $1+a$, where $a \in \mathcal{A}$.

LEMMA 3. $i_{*}: H_{1}(\tilde{V}) \rightarrow H_{1}(\tilde{X})$ is one-to-one.

Proof. Since $H_{1}(\tilde{V})$ is a $\Lambda$-torsion-free module of rank $m-1$ (see, for example, [Sa, Lemma 2.5]), it will suffice to show that

$$
1 \otimes i_{*}: \Lambda_{0} \otimes_{\Lambda} H_{1}(\tilde{V}) \rightarrow \Lambda_{0} \otimes_{\Lambda} H_{1}(\tilde{X})
$$

is one-to-one where $\Lambda_{0}$ is the quotient field of $\Lambda$, and to do this, it will suffice to show that $1 \otimes i_{*}$ is onto and $\operatorname{rk}\left(H_{1}(\tilde{X})\right)=m-1$. The first part is easy; $\operatorname{cok} i_{*}=K$ is of type $L$ and hence $\Lambda$-torsion so that $\Lambda_{0} \otimes_{\Lambda} K=0$. Now $H_{1}(\tilde{X})$ depends only on $\pi$, so it is enough to look at any complex $P$ with $\pi_{1}(P) \approx \pi$. It is essentially a result of Kervaire [Ke, Theorem 3] that there exists a finite 3-complex $P$ with only one 0 -cell such that $\pi_{1}(P) \approx \pi$ and $H_{*}(P) \approx H_{*}(V)$. The Euler characteristic of such a 
$P$ is $1-m$; hence, the Euler characteristic of $\tilde{P}$ over $\Lambda$ is $1-m$. By Theorem A, $H_{2}(\tilde{P})$ and $H_{3}(\tilde{P})$ are of type $L$ and hence of rank zero. $H_{0}(\tilde{P}) \approx \mathbf{Z}$ and has rank zero. It follows that $H_{1}(\tilde{P}) \approx \pi^{\prime} / \pi^{\prime \prime} \approx H_{1}(\tilde{X})$ has $\operatorname{rank} m-1$.

We have, as a consequence of Lemma 3 , a short exact sequence

$$
0 \rightarrow H_{1}(\tilde{V}) \stackrel{i_{*}^{*}}{\rightarrow} H_{1}(\tilde{X}) \stackrel{j}{\rightarrow} K \rightarrow 0
$$

and $K$ is of type $L$. Let $T_{1}$ be the $\Lambda$-torsion submodule of $H_{1}(\tilde{X})$, and let $F$ denote $i_{*} H_{1}(\tilde{V}) \subseteq H_{1}(\tilde{X})$. Since $F$ is torsion-free, $T_{1} \cap F=\{0\}$.

LEMMA 4. $T_{1}$ is of type $L$.

Proof. It suffices to show that $j \mid T_{1}: T_{1} \rightarrow K$ is one-to-one. For if this is so, let $C=\operatorname{cok}\left(j \mid T_{1}\right)$. Then $\mathbf{Z} \otimes_{\Lambda} K=0$ implies $\mathbf{Z} \otimes_{\Lambda} C=0$. By Lemma $1, \operatorname{Tor}_{1}^{\Lambda}(\mathbf{Z}, C)=0$. By the long exact sequence of Tor, we get that $\mathbf{Z} \otimes_{\Lambda} T_{1}=0$ because $\mathbf{Z} \otimes_{\Lambda} T_{1}$ falls between the two (zero) modules $\operatorname{Tor}_{1}^{\Lambda}(\mathbf{Z}, C)$ and $\mathbf{Z} \otimes_{\Lambda} K$.

Suppose $t \in T_{1}$ such that $\left(j \mid T_{1}\right)(t)=0$. Then $t=i_{*}(x)$ for some $x \in H_{1}(\tilde{V})$. Thus, $t \in T_{1} \cap F=\{0\}$. Therefore, $t=0$ and hence, $j \mid T_{1}$ is one-to-one.

This completes the proof of Theorem $\mathrm{B}$. We may now use the characterization theorem for the polynomials of boundary links [Sa, Theorem 6.1] in the context of general links. The proof is the same.

\section{REFERENCES}

[BE] D. Buchsbaum and D. Eisenbud, What makes a complex exact?, J. Algebra 25 (1973), 259-268. [BE2] _ - Some structure theorems for finite free resolutions, Adv. in Math. 12 (1974), 84-139.

[Dw] W. Dwyer, Vanishing homology over nilpotent groups, Proc. Amer. Math. Soc. 49 (1975), 8-12.

[HW] P. Hilton and S. Wylie, Homology theory: An introduction to algebraic topology, Cambridge Univ. Press, Cambridge, 1960.

[Ke] M. Kervaire, Les noeds de dimensions supérieures, Bull. Soc. Math. France 93 (1965), 225-271.

[Li] S. Lichtenbaum, On the vanishing of Tor in regular local rings, Illinois J. Math. 10 (1966), 220226.

[Sa] N. Sato, Algebraic invariants of boundary links, Trans. Amer. Math. Soc. 265 (1981), 359-374.

[Sm] J. Smith, Acyclic localizations, J. Pure Appl. Algebra 12 (1978), 117-127.

[St] J. Stallings Homology and central series of groups, J. Algebra 2 (1965), 170-181.

[Sw] R. Swan, Projective modules over Laurent polynomials rings, Trans. Amer. Math. Soc. 237 (1978), 111-120. Department of Mathematics, Texas A\&M University, College Station, Texas
77840 Department of Mathematics, Texas A\&M University, College Station, Texas
7840 